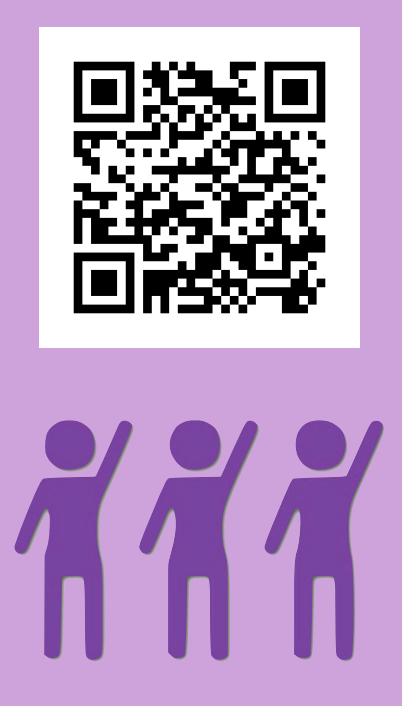

NÚMERO ESPECIAL

\title{
Percepções de mulheres sobre o momento do parto e a assistência obstétrica recebida
}

\author{
Miria BEnINCASA, Universidade Metodista de São Paulo \\ Adriana NAVARRo, Universidade Metodista de São Paulo \\ Neliane Lazarini de Sousa BETTIOL, Universidade Metodista de São Paulo \\ Maria Geralda Viana HELENo, Universidade Ibirapuera
}

\begin{abstract}
Este estudo teve como objetivo investigar a percepção de mulheres primíparas, com gestação de baixo risco sobre à assistência recebida e a experiência de trabalho de parto e pós-parto imediato. A amostra foi composta de 90 mulheres e os dados coletados por meio de entrevistas, que foram gravadas, transcritas e analisadas por meio da análise de conteúdo, que gerou cinco categorias. Três apriorísticas que são: - intervenções durante o parto; sentimentos vivenciados durante o trabalho de parto, pós-parto imediato e relacionamento com a equipe. E duas que emergiram da análise dos dados: violência obstétrica percebida e violência obstétrica não percebida. Os resultados demonstraram que a forma de a mulher perceber seu trabalho de parto e pós-parto imediato está intimamente associada ao modelo de assistência oferecida durante este processo. As participantes que optaram pelo Parto Humanizado revelaram sentimentos de gratidão, superação e transformação positiva. Este fato não foi verificado em nenhum outro modelo de assistência. Aquelas que experienciaram a Cesárea Eletiva ou Violência Obstétrica não vivenciaram o trabalho de parto e manifestaram afeto em relação à equipe apenas durante o momento de encontro com o bebe. Estas mulheres que foram vítimas de Violência Obstrética relataram indignação, revolta e desejo de vingança. Sugere-se estudos longitudinais que investiguem os prejuízos e benefícios destas manifestações afetivas intensas na identidade materna e no vínculo com o bebê.
\end{abstract}

PALAVRAS-CHAVE: Assistência obstétrica. Parto. Violência obstétrica. Humanização do parto. Pós-parto. 


\section{Introdução}

Entre as décadas de setenta e oitenta do século passado houve uma mobilização internacional no sentido de avaliar a assistência ao parto em todo o mundo e, o que estava em pauta era, entre outros aspectos, a excessiva medicalização do processo de parto sem evidências científicas e os elevados índices de cesárea em vários lugares do mundo (WHO, 2010). Em 1985, sob a coordenação da Organização Mundial de Saúde, realizou-se a "Conferência sobre Tecnologia Apropriada para o Parto" e essa reunião foi considerada um marco, tanto para a saúde pública, quanto para a defesa dos direitos da mulher e do bebê (PATAH; MALIK, 2011).

A Violência Obstétrica (VO) não está tipificada no Brasil, o conceito que será utilizado na presente pesquisa será o descrito pela Defensoria Publica do Estado de São Paulo (2013), que envolve: recusar a admissão em maternidade; impedir a presença do acompanhante de livre escolha da mulher durante todo o processo; realizar procedimentos que interfiram, causem dor ou dano físico; toda ação que gere "na mulher sentimentos de inferioridade, vulnerabilidade, abandono, instabilidade emocional, medo, acuação, insegurança, dissuasão, ludibriamento, alienação, perda de integridade, dignidade e prestígio"; dificultar o contato do bebê com a mulher após o parto, não oferecer alojamento conjunto mãe e bebê, caso haja possibilidade clínica; dificultar o aleitamento materno. Optou-se por esta referência por ser acessível às participantes e profissionais desta região pelo parâmetro de proximidade.

Apesar de uma Cesárea Eletiva (CE) sem indicação ser considerada por muitos autores como VO, optou-se neste estudo por não considerá-los similares. Definiu-se esta condução pelo fato de que os procedimentos que foram considerados $\mathrm{VO}$, inclusive aqueles que não foram reconhecidos como violência pelas mulheres, estão amplamente descritos tanto no discurso das participantes quanto nos documentos indicados como referência. As indicações de CE, entretanto, nem sempre foram acessíveis às pesquisadoras, não tendo parâmetros para considerá-las desnecessárias ou com indicação precisa. Estas avaliações poderiam ser realizadas apenas a partir de inferências não verificáveis. A título de exemplificação, uma participante com diagnostico de diabetes gestacional teve sua cesariana agendada com duas semanas de antecedência e a justificativa o risco aumentado com a manutenção da 
gestação. As pesquisadoras não têm elementos suficientes para avaliar se esta indicação foi necessária ou não. $O$ critério para inserção foi a eletividade da cirurgia, sendo excluída do conceito original de VO adotado para este estudo.

Para definir Parto Humanizado (PH) utilizou-se a referencia de Andrade e Lima (2014) que propõe: assistência baseada no protagonismo da mulher e na família; facilidade de acesso; acolhimento; incentivo à participação da mulher na tomada de decisões; priorizar o processo fisiológico, preterindo intervenções desnecessárias; uso adequado das tecnologias; práticas fundamentadas em evidências científicas.

$\mathrm{Na}$ realidade brasileira, as mulheres com gestaçao de baixo risco são desnecessariamente expostas a situações adversas e iatrogênicas que o excesso de tecnologia impõe, independente da sua situação socioeconômica (ANDREUCCI; CECATTI, 2011). Uma série de intervenções são realizadas como rotina que vão da cesarea desnecessárea à intervenções excessivas como episiotomia, gotejamento de ocitocina, amniotomia, entre outras. . As mulheres que têm poder aquisitivo mais elevado têm mais de $70 \%$ de chance de ser submetida a uma cesárea desnecessária. Aquelas que usam o serviço público têm 50\% de chance de intervenções de rotina prejudiciais ou de ser vítima de violência obstétrica (LEAL; PEREIRA; DOMINGUES; FILHA; DIAS; NAKAMURA-PEREIRA; BASTOS; GAMA, 2014).

Esta realidade gera impacto em outras áreas da vida da mulher e do bebê, que se estendem para além do momento do nascimento. A violência de gênero tem profundo impacto na autoestima e na manifestação de diagnósticos relacionados a transtornos de humor como depressão ou transtornos de ansiedade como Transtorno de Estresse Pós-Traumático (OKUDA; OLFSON; HASIN; GRANT; LIN; BLANCO, 2011). A Depressão Pós Parto é uma doença com grande incidência no mundo e com consequências negativas para mulheres, crianças e para família como um todo. No Brasil, a incidência gira em torno de $25 \%$ (THEME FILHA; AYERS; GAMA; LEAL, 2016).

Alguns estudos internacionais apontam maior frequência de Depressão Pós-Parto em mulheres que se submeteram a cesárea eletiva quando comparada com aquelas que vivenciaram parto vaginal (MATHISEN; GLAVIN; LIEN; LAGERLO, 2013). Mas, outros não indicam que a via de parto seja fator de risco para esta enfermidade 
(SWORD; LANDY; THABANE; WATT; KRUEGER; FARINE; FOSTER, 2011). Partindo destas premissas, o objetivo geral deste estudo foi investigar a percepção de mulheres sobre sua experiência durante o trabalho de parto e parto, inclusive a assistência que receberam da equipe de profissionais. Os objetivos específicos foram: verificar as percepções das mulheres durante o parto, analisar sentimentos vivenciados durante o trabalho de parto, parto e pós-parto imediato e investigar relacionamento com a equipe de assistência.

\section{Método}

Este estudo foi realizado entre os de 2015 e 2018, incluindo coleta e análise de dados. A equipe contou com duas professoras / orientadoras, três orientandas de mestrado, uma bolsistas de treinamento técnico e seis alunos de iniciação científica. Tratou-se de uma pesquisa exploratória, transversal, com análises quantitativas e qualitativas. Neste artigo será apresentado um recorte desta pesquisa maior, concentrando-se, predominantemente, nos resultados qualitativos. Segundo Minayo (2012, p. 622), a matéria prima deste tipo de estudo envolve experiências, sentimentos, vivências que se pretende "compreender, interpretar e dialetizar". O percurso analítico e sistemático, segundo a autora, se propõe a objetivar um conhecimento que se fundamenta em opiniões, crenças, representações, valores e relações humanas.

A Amostra, por conveniência, foi composta de 90 mulheres. Os critérios para a inclusão na amostra foram: a) ter passado pelo processo de parto há, pelo menos, 3 meses e, no máximo, 36 meses; b) ser o único filho (a), mesmo que tenha tido gestações anteriores; c) estar em uma relação conjugal estável, independente da orientação sexual; d) bebê não ser prematuro; e) ter tido uma gravidez de baixo risco, sem intercorrências; f) o bebê não apresentar diagnóstico de malformações ou síndromes genéticas (Tabela 1).

Tabela 1 - Caracterização da Amostra

\begin{tabular}{clcc}
\hline Dados de Identificação & Variáveis & Frequência & Porcentagem \\
\hline \multirow{2}{*}{ Raça } & branca & 55 & $61 \%$ \\
& preta & 09 & $10 \%$
\end{tabular}




\begin{tabular}{clll} 
& parda & 24 & $26 \%$ \\
& outros & 02 & $03 \%$ \\
\hline Idade & 18 a 19 & 03 & $03 \%$ \\
& 20 a 24 & 16 & $18 \%$ \\
& 25 a 30 & 27 & $30 \%$ \\
& 31 a 35 & 30 & $33 \%$ \\
& 36 a 40 & 09 & $10 \%$ \\
Escolaridade & 41 a 45 & 03 & $03 \%$ \\
& Acima de 46 & 02 & $03 \%$ \\
\hline & Médio incompleto & 04 & $03 \%$ \\
& Médio completo & 16 & $18 \%$ \\
& Superior incompleto & 14 & $15 \%$ \\
& Superior completo & 33 & $37 \%$ \\
& Pós-Graduação & 20 & $23 \%$ \\
& Mestrado & 04 & $04 \%$ \\
\hline Modelos de & Parto Humanizado (PH) & 46 & $51 \%$ \\
ao Parto & Cesárea eletiva (CE) & 30 & $34 \%$ \\
& Violência Obstétrica (VO) & 14 & $15 \%$ \\
\hline
\end{tabular}

Das 90 participantes avaliadas neste estudo (Tabela 1), no que se refere a raça, mais da metade se autodeclarou branca (61\%). Houve maior concentração de mulheres-mães entre 20 e 35 anos (81\%) e seus bebês com idade média 15 meses. A maioria (64\%) havia completado o ensino superior completo e $27 \%$ delas tinham pós-graduação latu ou strictu sensu. Metade (51\%) das mulheres considerou seu parto humanizado, um terço (33\%) relatou ter optado por cesárea eletiva e 15\% delas declararam ter sofrido VO durante o parto. Estes números referemse a como a mulher declara a assistência recebida no primeiro contato com as pesquisadoras, ou seja, 46 declararam ter experienciado um $\mathrm{PH}$, 30 optaram por CE e 14 foram vítimas de VO. No item "Resultados e Discussão”, será observada uma mudança nestes números, ou seja, após analise pormenorizada das entrevistas verificou-se que, entre as participantes que declararam a vivencia de $\mathrm{PH}$, houve práticas de $\mathrm{VO}$ ou agendamento de CE. Cada relato será descrito detalhadamente na "Categoria 1: intervenções durante o parto" 
No estudo original, ainda em processo de analise de resultados, buscava-se investigar fatores de risco e de proteção ao bem-estar da mulher durante o pós-parto, porém os resultados relacionados ao modelo de assistência levaram as pesquisadoras a investigar pormenorizadamente este aspecto e os resultados serão apresentados a seguir.

Os instrumentos utilizados foram a Entrevista Psicológica Semiestruturada e um questionário sociodemográfico composto de 25 questões, sendo a maior parte objetiva. As questões eram sobre dados pessoais, financeiros, ocupacionais, relacionadas ao período gestacional, ao parto, ao pós-parto e à saúde do bebê.

\section{Procedimentos}

Esta pesquisa foi aprovada no Comitê de Ética em Pesquisa da Universidade Metodista de São Paulo (CEP-UMESP): Número do Parecer: 896.875 de 26/11/2014, CAAE: 39023514.1.0000.5508. Depois da aprovação foram iniciados os contatos com as participantes por meio de grupos públicos de uma rede social. No momento em que a participante era contatada por telefone, e-mail ou mensagens, foi verificado se ela cumpria os critérios definidos para o estudo e agendouse uma visita. Nesta, inicialmente, apresentava-se os objetivos da pesquisa e o Termo de Consentimento Livre e Esclarecido e no caso de aceite era realizada a entrevista com duração média de 90 minutos. A coleta de dados foi feita no estado de São Paulo, basicamente, Grande São Paulo e Vale do Paraíba.

As entrevistas foram transcritas e foi realizada uma análise de conteúdo temático - categorial (OLIVEIRA, 2008). Optou-se por não usar nenhum tipo de tecnologia para estas análises, que foram realizadas e supervisionadas em um grupo de estudo para este fim. Compartilhando de impressões de Minayo (2012, p.625, 626), "sinto muita dificuldade em terceirizar, para tais dispositivos, a tarefa analítica" e complementa "toda a reflexão supõe a presença e o acompanhamento do pesquisador em cada passo do trabalho, num movimento ao mesmo tempo somativo e de superação da fase anterior".

Após leitura e releitura cuidadosa do material pela equipe de cinco psicólogas, foram identificados os conteúdos que pertenciam às categorias apriorísticas, já definidas previamente: intervenções durante 
o parto; sentimentos vivenciados durante o trabalho de parto, parto e pós-parto e relacionamento com a equipe. Outras categorias, entretanto, pela frequência e relevância, emergiram dos dados e foram inseridas nesta análise como: violência obstétrica reconhecida (VO1) e violência obstétrica não reconhecida (VO2). Esta categoria esclarece a Violência Obstétrica (VO) apresentada nos resultados e é apresentada em dois grupos, segundo a percepção da participante. Chamou-se de Violência Obstétrica 1 (VO1), quando esta experiência é vivida pela mulher como violenta e anunciada desta forma já no primeiro contato. Há percepção e reconhecimento do maltrato ou de procedimentos desnecessários ou dos direitos violados. No grupo que compõem a $\mathrm{VO} 2$, na descrição do parto, ha indicadores claros de inadequação segundo as descrições na literatura disponível (ANDRADE; LIMA, 2014; LEAL et al., 2014; BRASIL, 2014; ANDREUCCI; CECATTI, 2011), porém a VO não foi reconhecida pela participante. $\mathrm{Na}$ apresentação das categorias, além de descrever o conteúdo relatado pelas participantes, eventualmente foram inseridos alguns trechos emblemáticos relacionados ao tema. Para proteger a identidade das participantes, antes de cada fala há a indicação da letra P (participante) e o número de seu registro, segundo a ordem da coleta de dados.

\section{Resultados e Discussão}

\section{Categoria 1: intervenções durante o parto}

Das 90 participantes, 46 declararam ter vivido um parto humanizado. Mas, no discurso verificou-se a presença de intervenções que, segundo a literatura, são indicativas de violência obstétrica (VO) e Cesáreas Agendadas (ANDRADE; LIMA, 2014; LEAL et al., 2014; BRASIL, 2014). Entre as 46, 12 tiveram episiotomia e nenhuma foi consultada ou informada de que este procedimento seria feito, desconsiderando as diretrizes atuais de assistência ao parto, que desencorajam o uso rotineiro de episiotomia (CARROLI; MIGNINI, 2009; LEAL et al., 2014).

Duas tiveram alguém de sua livre escolha acompanhando apenas durante o período do expulsivo, sendo negado o direito de acompanhante durante o trabalho de parto e pós-parto, direito resguardado no Brasil pela Lei 11.108, de 7 de abril de 2005 (BRASIL, 2005), também chamada Lei do Acompanhante, que garante à 
parturiente a presença de um acompanhante, de sua livre escolha, durante o trabalho de parto, parto e pós-parto (BRASIL, 2014).

Uma participante passou por exame de toque de 10 profissionais diferentes, procedimento não recomendado pelas "boas praticas de assistência ao parto", mesmo para fins de aprendizagem (PARTO DO PRINCÍPIO, 2012; DINIZ, 2005). Onze tiveram sua cesárea agendada entre a $26^{\mathrm{a}}$ e $31^{\mathrm{a}}$ semana de gestação, para serem realizadas entre a $38^{\mathrm{a}}$ e $39^{\mathrm{a}}$ semanas, apesar de todas serem de baixo risco, conforme definição da amostra no projeto e não haver indicação expressa pelo médico para esta cirurgia. Foi solicitado a estas participantes que trouxessem o prontuário médico e, em todos eles, o motivo descrito para o procedimento foi "cesárea a pedido" (ou outro termo similar), embora nenhuma confirme esta solicitação. Este procedimento que, a principio, era destinado a salvar vidas, atualmente, por seu uso indiscriminado, vem expondo mãe e bebê a riscos desnecessários e, por esse motivo, tornou-se assunto em pauta por parte de autoridades no Brasil e em vários países do mundo (WHO, 2010; BRASIL, 2014).

Observa-se que mais de uma das intercorrências acima foram realizadas na mesma participante. Entre as que tiveram episiotomia, também foi negado o direito de acompanhante, porém, ainda assim, elas consideraram a assistência humanizada. Abaixo serão apresentadas algumas falas emblemáticas sobre estes resultados apontados pelas participantes. (Po3) "Correu tudo bem, não houve intervenção, só o corte embaixo que tem que fazer senão o bebê não sai, né?”; (P14) “A minha indicação de cesárea foi pela miopia. Tenho um grau alto e o médico disse que se eu fizesse força poderia piorar". Nestes relatos, que representam tantos outros colhidos, observa-se a falta de informação sobre a episiotomia e sobre parto (PIMENTEL; OLIVEIRA-FILHO, 2016). As participantes acreditam que para a realização de um parto vaginal, invariavelmente, é necessário o corte na vagina e que ter miopia é uma indicação clara de cirurgia cesariana.

Para todas as participantes foi questionado se houve intervenções e, após a resposta negativa, investigou-se especificamente cada intervenção descrita pela Organização Mundial de Saúde (WHO, 2014). Ao ser perguntado sobre o corte embaixo, em 12 participantes a resposta passou a ser positiva ( $\left.\mathrm{P}_{23}\right)$ "sim, claro" ou ( $\left.\mathrm{P}_{32}\right)$ "ah, o corte sim". Entre as participantes que tiveram a cesárea agendada, as justificativas médicas variaram entre: (Po8) "não vamos correr risco de esperar", (P82) "você pode avisar todo mundo e deixá-los preparados 
para receber seu bebê e vir te visitar", (P88) "sua estatura baixa pode interferir no momento do parto", entre outros. Estes motivos, no entanto, não estavam expressos nos prontuários, lidos pelas pesquisadoras e devolvidos às mães. Verifica-se, novamente, que escolher, concordar, ou aceitar a intervenção realizada está relacionado à falta de informação sobre o parto (PIMENTEL; OLIVEIRA-FILHO, 2016), mesmo para estas mulheres com nível de instrução superior ao da população brasileira e com acesso a internet.

Se inicialmente o maior volume de participantes era de $\mathrm{PH}$ e o menor de VO, após analise qualitativa verificou-se que aquelas que experimentaram Parto Humanizado $(\mathrm{PH})$ estavam em menor número, sendo superadas, em volume, pela VO e pela CE (Tabela 2). Alguns dos eventos apontados indicam experiência de violência obstétrica segundo a literatura disponível (ANDRADE; LIMA, 2014; LEAL et al., 2014; BRASIL, 2014; FENECH; THOMSON, 2013), porém, a maioria não se sentiu desrespeitada ou com direitos violados. Vale ressaltar que as pesquisadoras não interferiram nas impressões iniciais das participantes. Houve o entendimento de que não haveria benefícios em informar que houve violência para aquelas que afirmaram ter tido uma experiência humanizada.

Tabela 2 - Caracterização das participantes segundo o modelo de assistência ao parto após análise qualitativa

\begin{tabular}{lcc}
\hline Parto & Frequência & Percentual \\
\hline Parto Humanizado & 19 & $21 \%$ \\
Cesárea eletiva & 41 & $46 \%$ \\
Violência Obstétrica & 30 & $33 \%$ \\
Total & 90 & $100 \%$ \\
\hline
\end{tabular}

\section{Categoria 2: sentimentos vivenciados durante o trabalho de parto, parto e pós-parto}

Identificou-se pelas experiências das participantes deste estudo a presença de sentimentos positivos e negativos simultâneos e ambivalentes, tanto durante o processo de trabalho de parto e parto como em momento posterior ao nascimento. Takacs, Seidlerova e Hoskovcova (2015), avaliando os sentimentos de mulheres durante o trabalho de parto verificaram que em todas as participantes esta 
ambivalência é presente, podendo haver predominância maior de sentimentos positivos ou negativos. Observando tais ambivalências entre as participantes deste estudo, por meio da análise de conteúdo, verificou-se maior incidência de sentimentos positivos entre as que vivenciaram $\mathrm{PH}$, maior neutralidade entre as que optaram por $\mathrm{CE}$ ou $\mathrm{VO} 2$ e intensos sentimentos negativos entre as que foram vítimas de VO1.

Como afetos presentes de maneira uniforme entre as participantes destaca-se o medo e ansiedade relacionados ao processo, seja ele cirúrgico ou não, confirmando a literatura (ROWLANDS; REDSHAW, 2012). Os sentimentos positivos, entretanto, predominaram durante o processo do trabalho de parto e parto, entre aquelas que se prepararam para o parto humanizado, quando comparados aos relatos das participantes que descreveram um parto cirúrgico. Esse fato pode estar relacionado a preparação para a vivência deste momento. Kaźmierczak et al., (2015) relatam que este sentimento de auto eficácia e satisfação com seu corpo e com o parto tem relação com a quantidade e qualidade das informações que receberam sobre este momento; com o apoio que recebem das pessoas próximas e da equipe e do quanto a rede de apoio confirma sua capacidade de lidar com a situação do parto. Takacs, Seidlerova e Hoskovcova (2015), acrescentam que uma assistência perinatal menos formal, mais próxima e o ambiente físico favorável podem favorecer o aumento de sentimentos positivos. Com relação ao ambiente, 15 participantes que vivenciaram um $\mathrm{PH}$ estavam em ambiente mais acolhedor: três, tiveram seus filhos em casa e 12 foram no hospital, mas o parto ocorreu em salas de pré-parto e não em centro cirúrgico. As participantes inseridas nos grupos de $\mathrm{VO}$ ou de $\mathrm{CE}$ tiveram seus partos no centro cirúrgico.

$\mathrm{O}$ medo da dor do parto também foi um tema muito frequente, principalmente entre as que experimentaram CE. Enquanto 70\% das participantes deste grupo afirmaram que o agendamento foi feito por indicação ou necessidade do médico, 30\% relatou que fez essa opção e, dentre as motivações, a dor do parto esteve frequente (P61:"não suporto dor, não posso me imaginar passando pelo parto", $\mathrm{P}_{77}$ : "ninguém precisa sentir dor mais, não faz sentido querer isso, é muito primitivo.). Estes resultados corroboram achados da literatura nos quais há evidência de que o medo da dor conduz algumas mulheres a tomarem uma decisão de não terem mais filhos ou optarem por uma $\mathrm{CE}$, mesmo sem indicação clara (FENECH; THOMSON, 2013). Apesar de o pedido 
da mãe dever ser considerado (TOOHILL; FENWICK; GAMBLE; CREEDY; BUIST; TURKSTRA; RYDING, 2014), há evidências do quanto a $\mathrm{CE}$ em gestantes de baixo risco pode gerar mais prejuízo do que benefício à mãe e ao bebê (CLARA; MIKAEL, 2013; KOLOKOTRONI; MIDDLETON; GAVATHA; LAMNISOS; PRIFTIS; YIALLOUROS, 2012). Há um consenso entre autores que estudaram o medo do parto a indicar melhores desfechos caso a equipe de pré-natal ajude a gestante a lidar com este sentimento (FENECH; THOMSON, 2013). Outros autores sugerem intervenções grupais preventivas, para que o tema seja abordado e esclarecido por profissionais devidamente atualizados (TOOHILL et al. 2014).

Por outro lado, há autores (MORAES; MATTOS; MATÃO; MARTINS; NASCIMENTO, 2016) que desmistificam a crença da sociedade com o paradigma de que a dor física gera apenas resultados insatisfatórios. Há estudos em que a vivencia da dor, com ajuda da equipe e da rede de apoio, pode ser transformada e reconhecida como algo positivo, relacionado à superação e ao empoderamento (MORAES et al, 2016; TOOHILL et al., 2014). Entre as participantes deste estudo que vivenciaram o $\mathrm{PH}$ a experiência da dor coincide com os achados dos autores supracitados. Todas afirmaram sentir dor durante o trabalho de parto e parto. Algumas com intensidade próxima ao insuportável e outras de forma tolerável, no entanto, este registro não está, durante o discurso, associado a sentimentos negativos. Consideraram-se (Po2, Po6) "poderosas", superando obstáculos e vencedoras. Como os relatos de algumas das participantes desta pesquisa: (Po9) "depois de ter parido, sei que sou capaz de qualquer coisa"; (P12) "me senti uma leoa, capaz de tudo o que eu quisesse", (P16) "me acho poderosa, consegui, eu e ela (a filha)". Ryff e Singer (2003) ressaltam que a vida oferece naturalmente situações de crise, medo e ansiedade e que os indivíduos desenvolvem habilidades para o enfrentamento dessas adversidades, superando e possibilitando determinar consequências e transformações significativas para o funcionamento mais sadio do sujeito.

Velho, Santos e Collaco (2014), comparando mulheres que passaram por experiência tanto de parto normal humanizado como o parto cirúrgico obtiveram resultados parecidos com os do presente estudo. Assim, os resultados encontrados com relação ao parto humanizado se associavam a: busca de informações, sentir-se acompanhada, superação, aprendizado, realização, ou seja, um saldo positivo, acompanhado de bem-estar geral pela experiência. Esse 
discurso expressa o que Velho, Santos e Collaco (2014) chamam de "florescimento". As participantes concluíram a experiência compreendendo o processo natural do parto como um modo de vida que foi adaptado para à chegada do filho. Vivenciando o momento com extrema emoção, bom humor, esperança, resiliência e otimismo, atribuindo mais sentimentos positivos ao momento do parto, do que sentimentos negativos (SCORSOLINI-COMIN, 2012).

Foi possível verificar no trabalho, um grande contraste de sentimentos referente o momento do parto nas mulheres que vivenciaram um $\mathrm{PH}$, em comparação aos aspectos presentes no discurso das mulheres que realizaram a cirurgia cesariana (CE). Estas relataram mais sentimentos negativos do que positivos. A experiência do parto, para essas participantes, ficou marcada como um evento, descrito por algumas delas, como: (P58) "normal"; (P61) "pouco realizador"; (P61) "frio, sem emoção"; (P63) "passivo"; (P71) "solidão"; (P71) "o pior dia da vida"; (P77) "uma intervenção necessária"; (P77) "um meio para ter meu filho nos braços". Observa-se que as experiências se encontram em um espectro entre pouco significativo a ruim. As associações positivas nesta via de parto referiram-se, especificamente, ao momento de ver o (a) filho (a) ou ao apoio do (a) companheiro (a): (P61) "ele ficou o tempo todo do meu lado, viu o filho antes de mim e me contou"; (P65) "ele ficou emocionado quando o nenê saiu e eu me emocionei com ele"; ( $\left.\mathrm{P}_{71}\right)$ "ainda bem que ele estava comigo, me sentiria muito sozinha se não tivesse".

Carneiro, Santos e Souza (2018) informam que as mulheres que vivenciaram uma CE têm uma falsa ideia de parto respeitoso e de que a equipe tende a ser vista com capacitada e competente. Acrescentam informando que, ao contrário de um parto respeitoso, essa via de parto, quando eletiva e sem indicação clara, tira o protagonismo da mulher e transfere para a equipe, mantendo-a passiva e objeto de assistência, retirando-lhe o lugar de sujeito desta experiência. Neste estudo, os achados foram semelhantes, a experiência do parto foi um procedimento médico, pouco afetivo e impessoal.

Entre as participantes que foram vítimas de VO 1 (14 participantes), houve maior referência a sentimentos negativos. Relataram: medo, abandono, solidão, isolamento, terror, humilhação, negligencia descaso. As situações vivenciadas como violentas foram descritas em detalhe: (P41) "pedi para que não me cortasse e ela cortou sem anestesia"; (P44) "me mandou calar a boca várias vezes, mas eu 
estava com dor, tinha vontade de gritar (...) mas eu só chorava"; ( $\left.\mathrm{P}_{51}\right)$ "pedi para me darem anestesia, eu não estava aguentando mais (...) e a enfermeira me chamava de fraca". A experiência de um parto traumático, quando repleto de sentimentos negativos, tais como os vivenciados pelas participantes do grupo VO1 aumenta o risco de baby blues, depressão, Transtorno de Estresse Pós-Traumático, pensamentos suicidas e intensos sentimentos de perda e sofrimento, que podem ser estendidos aos bebês e suas famílias (FENECH; THOMSON, 2013).

A participação nas decisões, a experiência de ser respeitada e a empatia da equipe estão relacionadas a maiores índices de satisfação com o parto (TAKACS; SEIDLEROVA; HOSKOVCOVA, 2015; DIAZTELLO, 2016 ) e necessariamente as participantes de VO tendem a relatar maior índice de sentimentos negativos. Kaźmierczak, Gierszewska, Mieczkowska, Gebuza e Banaszkiewicz, (2015) sustentam que as vivencias positivas e negativas durante a gestação e o parto podem contribuir para uma alteração do humor no puerpério. Diaz-Tello (2016), acrescenta que experiências negativas tem impacto na vinculação com o bebê e na relação com a própria maternidade, estendendo estes prejuízos para além do momento do parto.

\section{Categoria 3: relacionamento com a equipe}

Observou-se, predominantemente, dois aspectos ao comparar as participantes que experienciaram o $\mathrm{PH}$ e aquelas que vivenciaram $\mathrm{CE}$, são eles: sentimentos positivos ou negativos direcionadas à equipe e segurança/insegurança pela quantidade de informações oferecidas pela mesma. As que tiveram $\mathrm{PH}$ revelaram segurança, vínculo e gratidão pelos cuidadores e pelo apoio fornecidos durante o processo. Foi considerado de extrema importância, entre estas participantes, o suporte profissional e social, que se estendia aos familiares e foi promovido pela equipe durante o trabalho de parto e parto. Kaźmierczak, et al. (2015) relatam que o suporte social e a inclusão da rede de apoio em situações difíceis auxiliam o indivíduo no enfrentamento das adversidades e inconstâncias da vida cotidiana, como foi identificado neste estudo. $\mathrm{O}$ indivíduo que recebe um suporte atencioso tem mais possibilidade de sentir-se equilibrado mesmo diante de obstáculos (KAŹMIERCZAK et al., 2015; MORAES et al., 2016; TAKACS; SEIDLEROVA; HOSKOVCOVA, 2015). Quanto aos sentimentos de gratidão e carinho, amplamente apontados pelas participantes do grupo de $\mathrm{PH}$, 
Kaźmierczak et al. (2015) ressaltam que o indivíduo que tem seus limites respeitados e possui constantemente o acolhimento de suas necessidades, cria vínculos e encontra satisfação na qualidade de suas vivências. Takacs, Seidlerova e Hoskovcova, (2015), acrescentam que uma assistência perinatal mais informal e empática, além do ambiente físico aconchegante podem favorecer o aumento de sentimentos positivos em relação a equipe e é potencializado pela permissão da presença de familiares durante o processo. Os autores informam que, quando a equipe insere a mulher no lugar de protagonista e viabiliza a liberdade de movimentos e expressão, sentimentos de gratidão emergem. Estes resultados são amplamente verificados nas entrevistas das participantes que vivenciaram o $\mathrm{PH}$.

Entre as mulheres que passaram pela CE houve predominância dos sentimentos de indiferença, decepção ou frustração com a equipe e com o processo. Quando relatam positividade indicam a competência técnica. Referem-se a equipe como profissionais que fizeram seu trabalho e que (P61) "não há sentido em manter vinculo afetivo"; ( $\mathrm{P}_{58}$ ) "a relação foi profissional, comprei um serviço e eles fizeram o que tinham que fazer". Velho et al. (2014), ao realizar uma revisão integrativa sobre a vivência do parto normal e do processo cirúrgico, também verificaram que a aquisição de informações durante o parto sobre o que está ocorrendo e quais os procedimentos que serão feitos tendem a gerar sentimentos de confiança na parturiente, independentemente do tipo de parto. Estes dados são compartilhados por outros autores nacionais e internacionais (TAKACS; SEIDLEROVA; HOSKOVCOVA, 2015; KAŹMIERCZAK et al.; BANASZKIEWICZ, 2015; MORAES et al., 2016). Estes procedimentos, tais como, informar sobre o processo de parto, sobre o que esperar, sobre a realização de intervenções, entre outros, foram negligenciados pelas equipes das participantes de CE e VO.

Entre as que estão no grupo de VO1 foram ouvidos relatos como: (P44) "estão sendo todos processados", (P48) "roubaram meu parto", (P55) "vão pagar por tudo que me fizeram passar"; (P57) "a única coisa boa disso tudo foi meu filho". Entre as que pertencem ao grupo de VO2, os afetos se assemelham ao do parto cirúrgico. Referem-se a uma relação profissional, que envolve poucos afetos e os relatos foram: (Po5) " $f o i$ como comprar um carro, a gente fica feliz no final, mas não lembra da cara do vendedor"; (P18) "fizeram o que tinham que fazer". A violência durante o parto pode gerar prejuízos importantes para a mulher, o bebê 
e a família (KAŹMIERCZAK et al., 2015) e, diferente das assistências CE e $\mathrm{VO}_{2}$, há predominância de sentimentos negativos e agressividade dirigida à equipe. Para Simpson e Catling (2015) há necessidade urgente de reduzir o risco das mulheres experimentarem o parto como um evento traumático. Justificam essa informação apontando os efeitos nocivos e as implicações negativas a longo prazo para mulheres e suas famílias.

\section{Categoria 4: Violência Obstétrica reconhecida (VO1) e não reconhecida (VO2)}

Entre as 14 participantes que indicaram a experiência de VO já no primeiro contato, verificou-se tanto procedimentos dolorosos sem consentimento, quanto humilhações, negligencia e abandono. Considerando os procedimentos, todas passaram por episiotomia sem serem informadas, seis delas não estavam anestesiadas durante o procedimento e cinco pediram para que não fosse feita assim que perceberam a intenção do profissional em realizá-la. A manobra de Kristeller foi realizada e descrita com detalhes em 07 (50\%) destas participantes. Em uma delas, a profissional posicionou o braço do parceiro (marido) para a realização da manobra.

Ao relatarem as humilhações, negligencia e abandono elas descrevem falas do tipo: ( $\left.\mathrm{P}_{42}\right)$ "se gritar não vou te atender", (P45) "cala a boca, deixa eu fazer o meu trabalho", (P46) "quem manda aqui sou eu, vou fazer episiotomia sim", (P49) "fica sozinha pra se acalmar, não precisa de anestesia". Todas as participantes foram conduzidas à ocitocina de rotina, duas se negaram a receber o "sorinho". As 14 participantes declaram-se envolvidas com instituições governamentais ou não governamentais de combate a violência de gênero ou, especificamente, à violência obstétrica. A maioria se declara militante (12 participantes), outras utilizam de serviços de assistência jurídica ou psicoterapia (6 participantes). Quatro participantes, além de militarem, utilizam os serviços jurídicos ou psicoterapêuticos.

A literatura mostra (OKUDA et al., 2011) que a violência de gênero gera consequências devastadoras na vivencia afetiva das mulheres por muito tempo. Fenech e Thomson (2013) e Simpson e Catling (2015) revelam que um parto traumático e angustiante pode ter um profundo impacto psicológico. Neste momento as mulheres experimentam intensas respostas negativas em relação a si mesmas e aos 
outros que podem se estender por anos. Tais vivências negativas impactam em muitos aspectos da vida podendo levar a manifestações cognitivas, comportamentais, sociais e psicológicas. Nas participantes deste estudo verifica-se uma tendência de buscar alternativas para lidarem com estas ocorrencias, sejam através de tratamentos psicológicos, por apoio jurídico ou pela militância contra a violência de gênero.

Outro aspecto relevante nos relatos é o envolvimento com organizações não governamentais voltadas ao enfrentamento da violência contra mulher como mecanismo compensatório e de superação da experiência traumática. Estudos nacionais (MENEZES et al., 2014) abordam a escassez de articulação intersetorial e de capacitação profissional para lidar com as questões relacionadas à violência e a encaminhamentos satisfatórios, levando à vitima a buscar recursos para além do circuito formal brasileiro. A militância, neste caso, torna-se uma alternativa eficaz.

Leal et al. (2014) mostram que uma entre quatro mulheres brasileiras relata ter passado por situações violentas durante o momento do parto. Há a hipótese, ainda naquele estudo, de que estes índices tenham sido subestimados, pois, uma quantidade significativa de mulheres não tem consciência de que não receber esclarecimentos sobre o que será feito com seu corpo, não fornecem consentimentos sobre determinadas intervenções, ter seu parto acelerado por conveniência profissional ou recebem indicações desnecessárias de cesarianas, se constituem, violência obstétrica (ANDRADE et al. 2014). Essa categoria, que emergiu dos dados na presente pesquisa ratifica essa hipótese. Estas 16 mulheres que compõem o grupo $\mathrm{VO} 2$ não tinham e ainda não tem nenhuma consciência de que as práticas que vivenciaram durante o trabalho de parto e parto não são recomendadas e, em alguns casos, viola seus direitos (SIMPSON; CATLING, 2015). Durante a descrição, os procedimentos relatados na "Categoria 1: intervenções durante o parto" foram amplamente realizados, entre as participantes que compõem o grupo de $\mathrm{VO} 2$ (16 participantes), principalmente episiotomia (12 participantes), manobra de Kristeler (4 participantes), impedimento da entrada de um acompanhante durante o trabalho de parto (2 participantes) e múltiplos exames de toque realizados por profissionais diferentes a fim de aprendizagem e sem consentimento prévio (14 participantes, sendo que uma, foi tocada por 10 médicos diferentes sem intervalos). Todas as 16 participantes do grupo VO2 receberam ocitocina 
de rotina e nove delas não receberam informações sobre os motivos pelos quais estavam recebendo a medicação. Observa-se que, em uma mesma participante, mais de um procedimento ocorreu, por exemplo: Manobra de Kristeller, episiotomia e ocitocina sintética de rotina; episiotomia, ocitocina sintética e impedimento do acompanhante. Não há, nesta categoria, como inserir trechos dos relatos que representem este grupo de participantes, visto que não reconhecem tais vivencias como violentas.

\section{Considerações Finais}

O objetivo deste estudo foi investigar a percepção de mulheres sobre sua experiência de trabalho de parto e parto. As coletas foram realizadas a partir do pós-parto imediato, até o pós-parto remoto (após o $45^{\circ}$ dia de nascimento do bebê, até seu $36^{\circ}$ mês de vida). A seleção da amostra incluiu vários critérios rigorosos com o objetivo de destacar os afetos e as percepções destas mulheres durante o trabalho de parto e parto, buscando eliminar outros fatores estressores que poderiam interferir na percepção sobre estas experiências.

Os objetivos da pesquisa foram atingidos, porém, a experiência positiva ou negativa do trabalho de parto e parto mostrou-se associada, prioritariamente, á qualidade da assistência recebida e não ao modelo de assistência, especificamente. Esta observação fica evidente quando as mulheres relatam às pesquisadoras ter experienciado um $\mathrm{PH}$ e, posteriormente, nas entrevistas, verificam-se procedimentos reconhecidos como VO, tais como: restrição de acompanhante, manobra de Kristeller, ocitocina sintética e episiotomia de rotina.

A literatura sobre psicologia do ciclo-gravídico-puerperal referenciada neste estudo indica um período de fragilidade e de risco aumentado quanto à saúde mental. Os resultados apontam que a qualidade da assistência durante este período, especificamente, no trabalho de parto e parto, tem impacto duradouro nas mulheres avaliadas. A idade media dos bebês no momento da avaliação foi de 15 meses e o sofrimento pela VO1, por exemplo, foi indicado através de choro e expressões de raiva. Por outro lado, aquelas que experienciaram um $\mathrm{PH}$ com uma equipe encorajadora, próxima e respeitosa, contam com orgulho, mesmo um ano depois do parto, sobre seus sentimentos de superação, potência e sentimentos de gratidão, de superação de transformação. 
Esta investigação demonstrou que a forma de a mulher perceber seu trabalho de parto e parto está intimamente associada ao modelo de assistência oferecido durante esse processo. As participantes que optaram pelo $\mathrm{PH}$ revelaram sentimentos de gratidão, de superação de transformação, o que não foi verificado em nenhum outro modelo de assistência. Aquelas que experienciaram a $\mathrm{CE}$ não vivenciaram o trabalho de parto e não consideraram o momento do parto relevante. Ressaltam como gratificante o encontro com o bebe. Os afetos predominantes relacionados ao momento do parto, quando apresentados, foram indiferença, solidão e o que chamaram de "frieza". As mulheres que foram vítimas de $\mathrm{VO}$ foram subdivididas em dois grupos: VO1, as que reconheceram a violência quando ocorreu e VO2, as que não identificam a violência apesar do relato indicar procedimentos e ações compatíveis com VO segundo o referencial adotado neste estudo (Defensoria Pública do Estado de São Paulo, 2013). As do grupo VO1 relataram indignação, revolta e desejo de vingança. As 14 mulheres que integraram este grupo choraram durante a entrevista e vinham buscando suporte em organizações não governamentais de apoio à mulher, tais como, entidades que ofereçam serviços psicológicos ou jurídicos, movimentos sociais contra a violência de gênero, entre outras. As integrantes do $\mathrm{VO} 2$, tanto no relacionamento com a equipe, quanto aos afetos predominantes, demonstraram uma percepção deste momento semelhante às relatadas pelas participantes de CE.

O pós-parto, principalmente de primeiro filho, é um período de transição e de adaptação desta mulher às demandas do bebê; à amamentação; à fisiologia corporal; à organização doméstica e familiar; entre outros. Partindo destas considerações, estes resultados geraram alguns questionamentos, tais como: os afetos vivenciados neste momento terão impacto na vida emocional e relacional desta mulher para além do pós-parto? Se a chegada de seu filho gerou tanta dor, esta experiência influenciará a forma de recebê-lo? Se a entrada no universo da maternidade é sentida como sofrida, será possível ressignificar o exercício da maternidade de modo a sentir-se empoderada para realizar esse papel? Por outro lado, a percepção de superação, associada ao sentimento de gratidão, proporcionados por esta experiência contribuem para um exercício da maternidade mais satisfatório? Esta pesquisa não responde a estas questões, mas as lança, sugerindo a necessidade de novos estudos longitudinais, que investiguem os prejuízos e benefícios destas vivencias na relação da mulher com ela mesma e com seu corpo; a 
confiança nas suas capacidades; o exercício da maternidade; desejo (ou falta deles) por mais filhos; na vida sexual, na relação com o bebê, com seu parceiro / sua parceira íntima (o).

Este estudo mostrou que o momento do parto pode contribuir para intensa mobilização de afetos positivos e negativos e esta experiência pode interferir, durante um longo período, na construção da sua identidade como mãe e sua adaptação aos papeis de companheira, cidadã, profissional, etc. Negligenciar o momento do parto e a relação entre recursos emocionais disponíveis nesta mulher e o suporte da equipe e da rede de apoio, significa desconsiderar o impacto desta vivencia na saúde materno-infantil. Esta pesquisa sugere investigações sobre o impacto dessa catarse na construção da identidade materna e dos laços com este bebê e seu desenvolvimento.

\section{Referências}

ANDRADE, M. A. C. LIMA, J.B.M.C. (2014). O Modelo obstétrico e neonatal que defendemos e como qual trabalhamos. Cadernos HumanizaSUS, v. 4., p 19-46, 2014. Disponível em: http:// www.red e h u manizasus.net/sites/default/files / caderno_humanizasus_v4_humanizacao_parto.pdf. Acesso em 20/08/2018.

ANDREUCCI, C. B.; CECATTI, J. G. Desempenho de indicadores de processo do Programa de Humanização do Pré-natal e Nascimento no Brasil: uma revisão sistemática. Cad. Saúde Pública, v. 27, n. 6, p. 1053-1064, 2011.

BRASIL. Lei n 11.108, de 7 de abril de 2005. Altera a Lei no 8.080, de 19 de setembro de 1990, para garantir às parturientes o direito à presença de acompanhante durante o trabalho de parto, parto e pós-parto imediato, no âmbito do Sistema Único de Saúde - SUS, 2005. Disponível em: http://www3.dataprev.gov.br/sislex/paginas/42/2005/11108.htm. Acesso em 20/08/2018.

BRASIL. Ministério da Saúde. HumanizaSUS 4: Humanização do Parto e do Nascimento. (2014). Disponível em: http://www.abenfomg.com.br/ $\begin{array}{lllllllllllllllllllll}\mathrm{s} & \mathrm{i} & \mathrm{t} & \mathrm{e} & / & \mathrm{a} & \mathrm{r} & \mathrm{q} & \mathrm{u} & \mathrm{i} & \mathrm{v} & \mathrm{o} & \mathrm{s} & / & \mathrm{o} & \mathrm{u} & \mathrm{t} & \mathrm{r} & \mathrm{o} & \mathrm{s} & /\end{array}$ 107_Caderno_Humanizasus_v4_HUMANIZA\%C3\%87\%C3\%83O_PAR TO_2014.pdf Acesso em 20/o8/2018. 
CARNEIRO, A. J. S.; SANTOS, G. O.; SOUZA, Z. C. S. N. Discurso de mulheres sobre a experiência do parto normal e da cesariana. Revista de Pesquisa: Cuidado é Fundamental Online, [S.l.], v. 10, n. 1, p. 233-241, jan . 2018. IS S N 2175-5361. ht tp:// dx.doi.org / 10.9789/2175-5361.2018.v10i1.233-241. Disponível em: <http:// www.seer.unirio.br/index.php/cuidadofundamental/article/view/5931>. Acesso em: 08/03/ 2018.

CARROLI, G.; MIGNINI, L. Episiotomy for vaginal birth. Cochrane Database of Systematic Reviews 2009, Issue 1. Art. No.:CDoo0081. Disponível em: file:///C:/Users/Miria_2/Downloads/ o912f5of42ec763333000000.pdf . Acesso em 20/o8/2018.

CLARA E.C.; MIKAEL, N. Cesarean section and development of the immune system in the offspring. American Journal of Obstetrics \& Gynecology. Expert Reviews Obstetrics, v. 208, n 4, p. 249-254, 2013.

DEFENSORIA PÚBLICA DO ESTADO DE SÃO PAULO (2013). Núcleo Especializado de Promoção e Defesa dos Direitos da Mulher e Associação Artemis. Disponível em: https://www.defensoria.sp.def.br/dpesp/ repositorio/41/Violencia\%20Obstetrica.pdf . Acesso em 20/08/2018.

DIAZ-TELLO, F. Invisible wounds: obstetric violence in the United States. Reproductive Health Matters, v. 24, p. 56-64, 2016. Doi: 10.1016/j.rhm.2016.04.004

DINIZ C. S. G. Humanização da assistência ao parto no Brasil: os muitos sentidos de um movimento. Cienc Saude Coletiva. v. 10, n. 3, p. 627-37, 2005 .

FENECH, G.; THOMSON, G. Tormented by ghosts from their past': A meta-synthesis to explore the psychosocial implications of a traumatic birth on maternal well-being. Midwifery, v. 30, n. 2, 2013. http:// dx.doi.org/10.1016/j.midw.2013.12.004.

KAŹMIERCZAK, M; GIERSZEWSKA, M; MIECZKOWSKA, E.; GEBUZA, G.; BANASZKIEWICZ, M. Analysis of Chosen Variables Psychological Determining the Occurrence of Mood Disorders After Childbirth. Adv Clin Exp Med, v. 24, n. 6, p. 1051-1057, 2015. https:// dx.doi.org/10.17219/acem/27452. 
KOLOKOTRONI, O; MIDDLETON, N; GAVATHA, M.; LAMNISOS, D.; PRIFTIS, K. N.; YIALLOUROS, P. K. Asthma and atopy in children born by caesarean section: effect modification by family history of allergies - a population based cross-sectional study. BMC Pediatrics, 12:179, 2012. Disponível em: http://www.biomedcentral.com/content/pdf/ 1471-2431-12-179.pdf. Acesso em 20/08/2018.

LEAL, M. C.; PEREIRA, A. P. E.; DOMINGUES, R. M. S. M.; FILHA, M. M. T.; DIAS, M. A. B.; NAKAMURA-PEREIRA, M.; BASTOS, M. H.; GAMA, S. G. N. Obstetric interventions during labor and childbirth in Brazilian low-risk women. Cadernos de Saúde Pública, v. 30, Suppl. 1, p. S17-S32, 2014. Disponível em: http://www.scielo.br/scielo.php? pid $=$ So102-311X2014001300005\&script $=$ sci_arttext\&tlng $=$ en. Acesso em 26/09/2018.

MATHISEN, S. E.; GLAVIN, K.; LIEN, L.; LAGERLO, P. Prevalence and risk factors for postpartum depressive symptoms in Argentina: a crosssectional study. Int $J$ Womens Health; v. 21, n. 5, p. 787-793, 2013. https://dx.doi.org/10.2147/IJWH.S51436 .

MENEZES, P. R. M.; LIMA, I. S.; CORREIA, C. M.; SOUZA, S. S.; ERDMANN, A. L.; GOMES, N. P. (2014). Enfrentamento da violência contra a mulher: articulação intersetorial e atenção integral. Dossiê Violência: questão de interface entre a saúde e a sociedade, Saúde \& Sociedade, v. 23, n. 3, p. 778-786, 2014. https://doi.org/10.1590/ So104-12902014000300004

MINAYO, M. C. S. Análise qualitativa: teoria, passos e fidedignidade. Ciência \& Saúde Coletiva, v. 17, n. 3, p. 621-626, 2012. https:// dx.doi.org/10.1590/S1413-81232012000300007

MORAES, A. P.; MATTOS, D. V.; MATÃO, M. E. L.; MARTINS, C. A.; NASCIMENTO, J. P. A dor do parto: percepção de mulheres que pariram no domicílio. Rev enferm UFPE, v. 10, Supl. 5, p. 4176-81, 2016. DOI: 10.5205/reuol.9284-81146-1-SM.1005sup201602. Disponível em: https://periodicos.ufpe.br/revistas/revistaenfermagem/article/view/ 11161. Acesso em 20/08/2018.

OKUDA, M.; OLFSON, M. P. H.; HASIN, D.; GRANT, B.F.; LIN, K.H.; BLANCO, C. Mental Health of Victims of Intimate Partner Violence: Results From a National Epidemiologic Survey. Psychiatric Services, v. 
62, n. 8, p. 959-962, 2011. https://dx.doi.org/10.1176/ps. 62.8.pss6208_0959

OLIVEIRA, D. C. Análise de conteúdo temático-categorial: uma proposta de sistematização. Rev. enferm. UERJ, v. 16, n. 4, p. 569-576, 2008. Disponível em: http://www.facenf.uerj.br/v16n4/v16n4a19.pdf . Acesso em 26/09/2018.

OliveirA, A. A. A.; ReSSTEL, C. C. P.; JUSTO, J. S. Desamparo Psíquico Na Contemporaneidade. Revista de Psicologia da UNESP, v. 13, n. 1, p. 21-32, 2014. Disponível em: http://pepsic.bvsalud.org/scielo.php? script=sci_arttext\&pid=S1984-90442014000100003\&lng=pt\&tlng=pt. Acesso em 26/09/2018.

PARTO DO PRINCÍPIO. Dossiê da Violência Obstétrica "Parirás com dor”, elaborado pela Rede Parto do Princípio para a CPMI da Violência Contra as Mulheres, 2012. Disponível em: http://www.senado.gov.br/ comissoes/documentos/SSCEPI/DOC\%20VCM\%20367.pdf. Acesso em 26/o9/2018.

PATAH, L. E. M.; MALIK, A. M. Modelos de assistência ao parto e taxa de cesárea em diferentes países. Revista de Saúde Pública, v. 45, n.1, 185-194, 2011. http://dx.doi.org/10.1590/So034-89102011000100021. Disponível em: http://www.scielo.br/scielo.php? script=sci_arttext\&pid=So034-89102011000100021\&lng =en\&nrm=iso >. Acesso em: 26/09/2018.

PIMENTEL, T. A.; OLIVEIRA-FILHO, E. C. Fatores que influenciam na escolha da via de parto cirúrgica: uma revisão Bibliográfica. Universitas: Ciências da Saúde, v. 14, n. 2, p. 187-199, 2016 DOI: 10.5102/ ucs.v14i2.4186. Disponível e m: https:// www.publicacoesacademicas.uniceub.br/cienciasaude/article/view/ 4186/3279. Acesso em 26/o9/2018.

ROWLANDS, I.J.; REDSHAW, M. Mode of birth and women's psychological and physical wellbeing in the postnatal period. $B M C$ Pregnancy and Childbirth, 2012, 12:138. Disponível em: http:// www.biomedcentral.com/1471-2393/12/138. Acesso em 26/o9/2018.

RYFF, C.D.; SINGER, B. Flourishing under fire: Resilience as a prototype of challenged thriving. In C.L.M.Keyes, \& J. Haidt (Eds.), Flourishing: 
Positive Psychology and the life well-lived (p. 15-36). 2003. Washington, DC: American Psychological Association.

SCORSOLINI-COMIN, F. Por uma nova compreensão do conceito de bem-estar: Martin Seligman e a psicologia positiva. Universidade Federal do Triângulo Mineiro, Uberaba-MG, Brasil. Paidéia, v. 22, n. 53 , p. 433-435. ht t p://dx.doi.org/10.159o/ So103-863X2012000300015. Disponível em: http://www.scielo.br/ scielo.php?script=sci_arttext\&pid=So103-863X2012000300015\&lng=en\&nrm=iso Acesso em: 26/09/2018.

SIMPSON, M.; CATLING, C. Understanding psychological traumatic birth experiences: A literature review. Women and Birth, v. 29, n. 3, p. 203-207, 2015. http://dx.doi.org/10.1016/j.wombi.2015.10.009.

SWORD, W. LANDY, C.K.; THABANE, L.; WATT, S.; KRUEGER, P.; FARINE, D.; FOSTER, D. Is mode of delivery associated with postpartum depression at 6 weeks: a prospective cohort study. BJOG: An International Journal of Obstetrics \& Gynaecology, v. 118, n. 8, p. 966-977, 2011. http://dx.doi.org/10.1111/j.1471-0528.2011.02950.x

TAKACS, L.; SEIDLEROVA, L.S.; HOSKOVCOVA, S.H. Social psychological predictors of satisfaction with intrapartum and postpartum care - whatmatters to women in Czech maternity hospitals? Open Med., v. 10, n. 1, p. 119-127, 2015. http://dx.doi.org/10.1515/ med-2015-0022 .

THEME FILHA, M. M.; AYERS, S.; GAMA, S. G. N. ; LEAL, M. V. C. Factors associated with postpartum depressive symptomatology in Brazil: The Birth in Brazil National Research Study, 2011/2012. J Affect Disord, v. 194, p. 159-67. 2016. http://dx.doi.org/10.1016/j.jad. 2016.01.020

TOOHILL, J.; FENWICK, J.; GAMBLE, J.; CREEDY, D. K.; BUIST, A.; TURKSTRA, E.; RYDING, E.L. A Randomized Controlled Trial of a Psycho-Education Intervention by Midwives in Reducing Childbirth Fear in Pregnant Women. BIRTH, v. 41, n. 4, 2014. http://dx.doi.org/10.1111/ birt.12136 .

VELHO, M.B; SANTOS, E.K.A; COLLACO, V.S. Parto normal e cesárea: representações sociais de mulheres que os vivenciaram. Rev. bras. enferm., v. 67, n. 2, p. 282-289, 2014. http://dx.doi.org/ 
10.5935/o034-7167.20140038. Disponível em: http://www.scielo.br/ pdf/reben/v67n2/0034-7167-reben-67-02-0282.pdf. Acesso em 26/o9/2018.

WHO - World Healt Organization. The Global Numbers and Costs of Additionally Needed and Unnecessary Caesarean Sections Performed per Year: Overuse as a Barrier to Universal Coverage, 2010. Disponível em: http://www.who.int/healthsystems/topics/financing/healthreport/ 30C-sectioncosts.pdf . Acesso em 20/08/2018.

WHO - World Healt Organization. Prevenção e eliminação de abusos, desrespeito e maus-tratos durante o parto em instituições de saúde. Declaração da Organização Mundial de Saúde, 2014. Disponível em: http: / / apps.who.int/iris/bitstream/10665/134588/3/ WHO_RHR_14.23_por.pdf e http://www.who.int/reproductivehealth/ topics/maternal_perinatal/statement-childbirth/en/. Acesso em 20/08/2018. 


\section{Women's Perceptions about the Moment of Birth and Obstetric Care Received}

ABSTRACT: This study aimed to investigate the perception of primiparous women with low-risk pregnancies about the care received and the experience of labor and delivery. The sample consisted of 90 women. Data were collected through recorded interviews, transcribed and interpreted through categorical content analysis. Four categories were created. Three aprioristics: 1) interventions during childbirth; 2) feelings experienced during labor, delivery and immediate postpartum and 3) relationship with the team. In addition, a fourth category emerged from the data: obstetric violence (VO), named as recognized obstetric violence (VO1) and unrecognized obstetric violence (VO2). The results showed that women's perceptions of labor and delivery are closely associated with the care model offered (during this process). Participants who opted to Humanized Childbirth $(\mathrm{PH})$ revealed feelings of gratitude, overcoming and transformation, which was not verified in any other model of care. Those who experienced Cesarean Elective (EC) or $\mathrm{VO}_{2}$ did not experience labor and did not express affection towards the team or the moment of delivery, only during the encounter with the baby. Women who were victims of $\mathrm{VO} 1$ reported outrage, anger and desire for revenge. Longitudinal studies are suggested to investigate the harms and benefits of these intense affective manifestations in maternal identity and bonding with the baby.

KEYWORDS: Obstetric assistence. Parturition. Obstetric violence. Humanizing delivery. Postpartum period.

Miria BENINCASA

Pesquisadora e orientadora dos Programas de Pós Graduação (M/D) em Psicologia da Saúde da Universidade Metodista de São Paulo e de Psicossomática (M) da Universidade Ibirapuera. Editora-Chefe da Revista Mudanças - Psicologia da Saúde. Tem experiência na área de Psicologia da Saúde, com ênfase em Programas de Atendimento Comunitário, institucional e hospitalar. As principais áreas de pesquisa são: 1. Gestação: Avaliação e assistência Psicológica à gestante/ casal e desenvolvimento de Programas de Pré-Natal Psicológico, Pré-Natal Coletivo

e Pré-Natal Integral; 2. Parto e Pós-parto: modelos de intervenção, avaliação, apoio e acolhimento à mulheres e familias em situação de pós parto; 3.Psicologia do Desenvolvimento de Bebês e crianças (primeiríssima infância). 


\section{Adriana NAVARRO}

Doutoranda em Psicologia da Saúde (Universidade Metodista de São Paulo), Mestre em Psicologia da Saúde (Universidade Metodista de São Paulo/ Instituto Superior de Psicologia Aplicada de Lisboa - ISPA). Especialista em Psicologia Hospitalar e da Saúde (CEPPS). Docente do curso de especialização em

Psicologia Hospitalar e da Saúde da Universidade de Taubaté. Psicóloga Clínica com atendimento em psicologia obstétrica e saúde materno infantil. Experiência em saúde pública; atendimento psicológico hospitalar e ambulatorial; educação

permanente em humanização; atendimento clínico; intervenção lúdica; saúde reprodutiva e psicoterapia breve. As principais áreas de pesquisa são: 1. Psicologia Obstétrica; 2. Humanização da assistência ao Parto e do Nascimento ; 3. Saúde da materno-infantil.

\section{Neliane Lazarini de Sousa BETTIOL}

Possui graduação em Psicologia pela Universidade Metodista de São Paulo (2013). Psicóloga clínica com atendimento de adultos e em psicologia obstétirca, saúde materno-infantil e parentalidade. Experiência em acompanhamento de pais de autistas e em psicoterapia breve operacionalizada. Mediadora de grupos de pré-natal psicológico e integral e grupos de pós parto. As principais áreas de pesquisa são: 1. Psicologia Obstétrica; 2. Humanização da assistência ao parto e nascimento; 3. Saúde da mulher.

\section{Maria Geralda Viana HELENO}

Graduação em Psicologia pelo Instituto de Ensino Superior Senador Fláquer (1978), mestrado em Psicologia da Saúde pela Universidade Metodista de São Paulo (1992) e doutorado em Psicologia Clínica pela Universidade de São Paulo (200o). Tem experiência na área de Psicologia com ênfase em Psicologia da Saúde. Atua e pesquisa nos seguintes temas: gravidez, parto e puerpério. Coordenadora do

Programa de Pós-Graduação em Psicologia - Psicossomática da Universidade Ibirapuera. Pós-doutorado em andamento na Universidade Federal de São Paulo.

Recebido em: 22/o1/2019

Aprovado em: 26/12/2019 